This is the post peer-review accepted manuscript of:

F. Guidi, A. Mariani, A. Guerra, D. Dardari, A. Clemente and R. D'Errico, "Indoor EnvironmentAdaptive Mapping With Beamsteering Massive Arrays," in IEEE Transactions on Vehicular Technology, vol. 67, no. 10, pp. 10139-10143, Oct. 2018.

doi: https://doi.org/10.1109/TVT.2018.2853657

The published version is available online at:

http://ieeexplore.ieee.org/stamp/stamp.jsp?tp=\&arnumber $=8405558 \&$ isnumber $=8492481$

C 2018 IEEE. Personal use of this material is permitted. Permission from IEEE must be obtained for all other uses, in any current or future media, including reprinting/republishing this material for advertising or promotional purposes, creating new collective works, for resale or redistribution to servers or lists, or reuse of any copyrighted component of this work in other works 


\title{
Indoor Environment-Adaptive Mapping with Beamsteering Massive Arrays
}

\author{
Francesco Guidi Member, IEEE, Andrea Mariani Member, IEEE, Anna Guerra Member, IEEE, \\ Davide Dardari Senior, IEEE, Antonio Clemente Senior, IEEE, Raffaele D'Errico Member, IEEE
}

\begin{abstract}
Beamsteering massive arrays have been recently proposed for indoor environment mapping in next $5 \mathrm{G}$ scenarios thanks to their capability to better penetrate materials with respect to current laser or vision-based systems. In the perspective of integrating radars in small portable devices, architectures based on non-coherent processing of raw measurements represent a viable solution to overcome the limitations of current indoor radio mapping techniques, which entail a too high processing or receiver complexity. In this paper we investigate the capability of low-complexity mobile radars, equipped with $\mathrm{mm}$-wave massive arrays, to adapt to the environment in order to reconstruct it, by adjusting a threshold with respect to the collected data and the radiation pattern. Results, corroborated by means of a measurement campaign, show the effectiveness of the proposed approach.
\end{abstract}

Index Terms-Massive Arrays, Beamsteering, Indoor Mapping, Adaptive Threshold.

\section{INTRODUCTION}

The joint use of millimeter-waves (mm-wave) and massive arrays technologies has recently shown the capability to pack a large number of antennas onto a small area, thus paving the way for a future integration in next generation portable devices (e.g. 5G) [1]-[3]. Thanks to the possibility to better penetrate materials than laser and vision-based systems, mm-wave massive arrays could be integrated into such systems. Towards this direction, devices moving in indoor environments could assist the user navigation even in scarce visibility conditions or could enable the creation of indoor maps, where Global Positioning System (GPS) fails, without exploiting signals coming from ad-hoc infrastructures [4]. Consequently, to enable the possibility to integrate such systems in relatively low-cost and low-power portable devices for personal radar applications, there is the need to reduce both the receiver and the algorithm complexity [1]. However, when limiting the array complexity for implementation constraints (e.g using a small number of phase quantization bits to combine signals [2]) the array radiation pattern is far from being laser-like and side-lobes are present [2], [5]. To counteract such an

F. Guidi, A. Clemente and R. D'Errico are with CEA, LETI, MINATEC Campus, 38054 Grenoble, France. They are also with Univ. Grenoble-Alpes, 38000 Grenoble, France. e-mail:\{antonio.clemente, raffaele.derrico\}@cea.fr

A. Mariani, A. Guerra and D. Dardari are with the Department of Electrical, Electronic, and Information Engineering "Guglielmo Marconi" - DEI, University of Bologna,Via Venezia 52, 47521 Cesena, ITALY. (e-mail: \{f.guidi, a.mariani, anna.guerra3, davide.dardari\}@unibo.it).

The work has been partially funded by the European Commission under H2020 project XCYCLE (grant nr. 635975), IF-EF Marie Curie project MAPS (grant nr. 659067) and IF-GF Marie Curie project AirSens (grant nr. 793581) undesired effect, several solutions have been tackled to guarantee high mapping performance [6]. Regarding techniques not accounting for massive arrays, the CLEAN approach has been usually considered robust in mitigating the image artifacts [7], [8]. For the considered mm-wave personal radar, such a technique cannot be exploited in practice due to the need of a high-complexity architecture to guarantee a large number of iterations and perfect alignment of signals [9].

Other works considered an adaptive scheme based on the generalized likelihood ratio test (GLRT) for detecting coherent pulses in presence of disturbances [10] and a side-lobe blanker, eventually provided by a single array element, to eliminate interference [11], [12]. Again, these solutions demand coherent receivers [13]. Thus current imaging techniques not exploiting massive arrays require an implementation complexity on a real hardware which would be unfeasible, especially at $60 \mathrm{GHz}$ [10], [12]-[15].

Nonetheless, state-of-the-art approaches based on the use of large antenna elements arrays usually do not exploit simple non-coherent architectures, apart from [16] where the receiver is replicated for each antenna. Thus, the procedure would result to be too much expensive when an extremely large number of antennas is employed, which is not in line with the requirements of our application.

Consequently, to assure mapping capabilities in low-cost and low-complexity portable devices, a single energy detector [1] with analog beamsteering massive arrays has been proposed, overcoming the need of digital chains that entail integrating expensive analog-to-digital converters [17]. The approach in [5] requires a preliminary estimation of the environment backscattering characteristics to properly work.

To move a step forward and overcome the shortcomings in [5] due to the need of a-priori map information, we propose an approach based on the following two phases: (i) a first noisethresholding phase to mask unwanted noise components; (ii) a second new thresholding phase to ameliorate the mapping, which is adaptive with respect to the environment as it compares measurements collected from different steering directions and takes advantage on the knowledge of the radiation pattern.

In summary, our new contributions with respect to the stateof-the-art are as follows.

- The proposal of a new adaptive scheme for indoor environment mapping, not requiring a high complexity processing (like, e.g., the CLEAN-inspired algorithms) that keeps the overall complexity as low as possible to ease the integration into a portable device [5], [18]; 
- The introduction of a new thresholding strategy able to adapt itself to the measured signals, overcoming the limitations present in [5] and mainly related to the need of having a-priori knowledge of the backscattering properties of the indoor environment;

- The performance assessment, using measurement data, of the considered architecture which is simpler than those requiring a higher complexity (i.e. coherent receivers) allowing the reconstruction of a map of the surrounding indoor environment with a portable device equipped with a massive array.

The rest of the paper is organized as follows. Sec. II describes the system model. Sec. III investigates the proposed adaptive thresholding technique, whose results are detailed in Sec. IV. Finally, conclusions are drawn in Sec. V.

\section{SignAl MODEL}

We now describe an ad-hoc signal model suitable for the system under investigation. In particular, the considered portable radar is based on the concept that a mobile device, equipped with a massive array, moves in an unknown indoor environment and for each position steers its beam towards different directions to reconstruct the surrounding scenario. The radar investigated exploits a monostatic configuration, i.e., the transmitter and receiver are co-located. For each steering direction $\theta_{b}$, the transmitter sends a train of $N_{\mathrm{p}}$ pulses and, for each pulse, it collects the backscattered response to detect the possible presence of targets and their distances with respect to the radar itself. ${ }^{1,2}$

To avoid the complexity entailed by coherent receivers, here we account for an energy detector. Let $N_{\text {steer }}$ be the number of steering directions during the scanning process where, for each steering direction, a generic interrogation signal composed of $N_{\mathrm{p}}$ pass-band pulses $p(t)$ of large bandwidth $W$ and central frequency $f_{\mathrm{c}}$ is transmitted

$$
g(t)=\sum_{l=0}^{N_{\mathrm{p}}-1} p\left(t-l T_{\mathrm{f}}\right)
$$

with $T_{\mathrm{f}}$ being the time frame chosen so that all signals backscattered by the environment are received before the transmission of the successive pulse, avoiding inter-frame interference. Since each pulse is backscattered by the surrounding targets, the received signal for the steering direction $\theta_{b}$ can be expressed as

$$
r\left(t, \theta_{b}\right)=\sum_{l=0}^{N_{\mathrm{p}}-1} g\left(t-l T_{\mathrm{f}}, \theta_{b}\right)+n(t)
$$

where $g\left(t, \theta_{b}\right)$ is the response to the transmitted pulse $p(t)$ at direction $\theta_{b}$ and with $n(t)$ being the additive white Gaussian noise (AWGN) with two-sided power spectral density $N_{0} / 2 .^{3}$

\footnotetext{
${ }^{1}$ Note that here we simplify the analysis to the case in which the beamsteering is performed over the plane defined by the azimuth angle $\theta_{b}$.

${ }^{2}$ Differently from laser-based systems, the beam can be electronically steered, thus avoiding mechanical components and easing the integration in small devices.

${ }^{3}$ For fixed $\theta_{b}$, we assume that the channel and noise characteristics are the same for each transmitted pulse.
}

The receiver section is the same as the one described in [5], where the collected signal is first filtered with an ideal bandpass filter (BPF) with center frequency $f_{\mathrm{c}}$. The filtered signal is denoted by

$$
y\left(t, \theta_{b}\right)=\sum_{l=0}^{N_{\mathrm{p}}-1} x\left(t-l T_{\mathrm{f}}, \theta_{b}\right)+z(t)
$$

where $x\left(t, \theta_{b}\right)$ and $z(t)$ are the filtered versions of $g\left(t, \theta_{b}\right)$ and $n(t)$, respectively. The energy receiver is chosen due to the complete uncertainty on the received waveform shape deriving from the a-priori ignorance on the surrounding environment. Energy is then evaluated for each time frame $T_{\mathrm{f}}$ after the transmission of each pulse by dividing the time frame into $N_{\text {bins }}=\left\lfloor T_{\mathrm{f}} / T_{\mathrm{ED}}\right\rfloor$ time slots (bins) of duration $T_{\mathrm{ED}}{ }^{4}$ and energy measurements are accumulated for each time bin over the $N_{\mathrm{p}}$ frames of the received signals. Such an operation is repeated for each steering direction, giving a $N_{\text {bins }} \times N_{\text {steer }}$ matrix $\mathbf{E}$, whose generic element at the $s$ th time bin and $b$ th steering angle is

$$
\begin{aligned}
e_{b s} & =\sum_{k=0}^{N_{\mathrm{p}}-1} \int_{(s-1)}^{s T_{\mathrm{ED}}} y^{2}\left(t+k T_{\mathrm{f}}, \theta_{b}\right) d t \\
& \simeq \frac{1}{2 W} \sum_{k=0}^{N_{\mathrm{p}}-1} \sum_{i=(s-1) N_{\mathrm{d}}}^{s N_{\mathrm{d}}}\left(x_{i}\left(\theta_{b}\right)+z_{i}^{(k)}\right)^{2}
\end{aligned}
$$

with $s=1,2, \ldots, N_{\text {bins }}$ and $b=1,2, \ldots, N_{\text {steer }}$, and $N_{\mathrm{d}}=$ $2 W T_{\mathrm{ED}}, z_{i}^{(k)}$ are for odd $i$ (even $i$ ) the samples of the real (imaginary) part, respectively, of the equivalent low-pass of $z\left(t+k T_{\mathrm{f}}\right), k=0,1, \ldots, N_{\mathrm{p}}-1$, taken at Nyquist rate $W$ in each interval $T_{\mathrm{ED}}$ and $\sigma^{2}=N_{0} W$ is the noise variance. In (4) we used the property $x\left(t+k T_{\mathrm{f}}, \theta_{b}\right)=x\left(t, \theta_{b}\right)$, so that $x_{i}\left(\theta_{b}\right)$ represents for odd $i$ (even $i$ ) the samples of the real (imaginary) part of the equivalent low-pass of $x\left(t, \theta_{b}\right)$, taken at Nyquist rate $W$ in each interval $T_{\mathrm{ED}}$.

In the following, we describe the proposed approach, which first accounts for a traditional thresholding phase (phase 1) and, successively, for a new thresholding phase (phase 2) to mask unwanted side-lobe contributions.

\section{THRESHOLD DESIGN}

\section{A. Phase 1: Noise Threshold Design}

The first step requires to mask unwanted noisy energy bins through the adoption of traditional detection approaches. In fact, energy bins in $\mathbf{E}$ belong to two main categories: onlynoise and useful (i.e., useful signal in addition to the noise) bins. Thus, at the output of phase 1, only the useful bins are kept and the information on the bin index $s$ and on the steering direction $\theta_{b}$ is gathered. To this end, we define a threshold based on the probability to wrongly classify a bin due to the receiver noise so that, in presence of only noise, i.e. $y\left(t, \theta_{b}\right)=$ $z(t)$, (4) can be written as

$$
e_{b s} \simeq \frac{1}{2 W} \sum_{k=0}^{N_{\mathrm{p}}-1} \sum_{i=(s-1) N_{\mathrm{d}}}^{s N_{\mathrm{d}}}\left(z_{i}^{(k)}\right)^{2} .
$$

\footnotetext{
${ }^{4}$ Note that $T_{\mathrm{ED}}$ must be chosen to accommodate most of the energy of the
} received pulse, i.e. $T_{\mathrm{ED}} \approx 1 / W$. 
In (5) we have the sum of the squares of $N=N_{\mathrm{p}} N_{\mathrm{d}}$ independent Gaussian random variables (RVs) which turns out into a central chi-square distribution, with $N$ degrees of freedom. A threshold-crossing event at the sth bin, that is, $\left\{e_{b s} \geq \xi_{b s}^{z}\right\}$, results in a single-bin probability of false classification (PFC) $p_{b s}^{(\mathrm{FC})}$ given by [5], [19]

$$
p_{b s}^{(\mathrm{FC})}=\tilde{\Gamma}\left(\frac{N}{2}, \frac{\xi_{b s}^{z}}{2}\right)
$$

with $\tilde{\Gamma}$ denoting the regularized gamma function [19]. The noise threshold $\xi_{b s}^{z}$ can be computed starting from a requirement on the global PFC $P_{\mathrm{FC}}^{\star}$ given by

$$
P_{\mathrm{FC}}^{\star}=1-\prod_{b=1}^{N_{\text {steer }}} \prod_{s=1}^{N_{\text {bins }}}\left(1-p_{b s}^{(\mathrm{FC})}\right) \approx N_{\text {bins }} N_{\text {steer }} \cdot p_{\mathrm{FC}}
$$

where we assume that all bins are statistically independent and $p_{b s}^{(\mathrm{FC})}=p_{\mathrm{FC}}$, with $p_{\mathrm{FC}} \ll 1$. Equation (7) translates into a required $p_{\mathrm{FC}}^{\star}$ per bin given by

$$
p_{\mathrm{FC}}^{\star} \approx \frac{P_{\mathrm{FC}}^{\star}}{N_{\mathrm{bins}} N_{\text {steer }}} .
$$

Note the threshold does not depend on the bin and steering indexes, i.e. $\xi_{b s}^{z}=\xi^{z}$, and it is set to keep the PFC due to the receiver noise to a desired value $P_{\mathrm{FC}}^{\star}$. Then, according to [19], we can write

$$
\xi^{z}=2 \sigma^{2}\left[\operatorname{Inv} \tilde{\Gamma}\left(\frac{N}{2}, \frac{P_{\mathrm{FC}}^{\star}}{N_{\text {bins }} N_{\text {steer }}}\right)\right]
$$

where $\operatorname{Inv} \tilde{\Gamma}(\cdot, \cdot)$ is the inverse gamma regularized function.

As shown in [5], by adopting the previously defined threshold, the system becomes sensitive to the presence of interference in the side-lobe direction. Thus, the technique in [5] has been proposed, relying on a-priori assumptions on the environment backscattering properties, and it could become limiting in real-time localization scenarios. To avoid the need of having any preliminary information on the environment, in the following we discuss a procedure that, starting from (9), allows to adapt the threshold to the measured signals.

\section{B. Phase 2: Adaptive Threshold Design}

In order to keep the overall complexity affordable, we define an adaptive threshold approach, which does not need any apriori information of the environment. Let consider the set

$$
\mathcal{B}=\left\{\mathcal{B}_{1}, \ldots, \mathcal{B}_{s}, \ldots, \mathcal{B}_{N_{\text {bins }}}\right\}
$$

whose generic element $\mathcal{B}_{s}$ contains the steering angle indexes of the energy bins $e_{b s}$ that have overcome the noise-threshold during the phase 1 for a given time bin index $s .{ }^{5}$ Moreover, we consider $\mathcal{B}_{s}$ to be arranged as

$$
\mathcal{B}_{s}=\left\{b_{1 s}, \ldots, b_{(l-1) s}, b_{l s}, \ldots, b_{\mathcal{L}_{s} s}\right\}
$$

so that the corresponding set of energies

$$
\mathcal{E}_{s}=\{\underbrace{e_{b_{1 s} s}, \ldots, e_{b_{(l-1) s} s}}_{\mathcal{E}_{(l-1) s}} \quad e_{b_{l s} s}, \ldots, e_{b_{\mathcal{L}_{s}} s}\}
$$

\footnotetext{
${ }^{5}$ The ranging information $d$ is related to $s$ in the form $d \approx s \cdot c T_{\mathrm{ED}} / 2$,
} with $c$ being the speed of light. is organized in decreasing order, i.e.,

$$
e_{b_{1 s} s}>\ldots>e_{b_{l s} s}>\ldots>e_{b_{\mathcal{L}_{s}} s}
$$

where $\mathcal{L}_{s}=\left|\mathcal{B}_{s}\right|$ is the cardinality of the set and varies according to $s$. Thus, we have the definition of $N_{\text {bins }}$ sets which can be empty or upper bounded by the number of steering directions $N_{\text {steer. }}$. For each distance, i.e., for each time index $s$, we can assume that if at least one bin is above the threshold $\xi^{z}, b_{1 s}$ likely corresponds to the steering direction where the target is intercepted and thus $e_{b_{1 s} s}$ will be considered at the output of phase 2.

Let also define the respective non-centrality parameter (NCP) vector given by

$$
\mathcal{N}_{s}=\left\{e_{b_{1 s} s}-\xi^{z}, \ldots, e_{b_{l s} s}-\xi^{z}, \ldots, e_{b_{\mathcal{L}_{s}} s}-\xi^{z}\right\}
$$

where each NCP element is the difference between the correspondent measured energy bin and $\xi^{z}$. To keep the algorithm complexity low, we design the threshold by accounting for the presence of a single target in the environment. ${ }^{6}$ Specifically, if $\mathcal{L}_{s} \geq 2$, for $l>1$ we consider the energy test

$$
e_{b_{l s} s} \stackrel{\mathcal{H}_{1}}{\underset{\mathcal{H}_{0}}{\gtrless}} \xi_{l s}^{x}
$$

where $\xi_{l s}^{x}$ is the threshold set in the phase 2 which expression will be hereafter derived. When $\mathcal{H}_{1}$ in (15) is satisfied, the corresponding energy bin $e_{b_{l s} s}$ is considered at the output of phase 2 in addition to $e_{b_{1 s} s}$. Specifically, we propose a method according to which, for the $s$ th time bin and the $l$ th steering direction, the threshold in (15) accounts only for the subset $\mathcal{E}_{(l-1) s}$ including the previous $l-1$ bins of the set in (12). Such an operation is performed in order to check if a component has been intercepted in a side-lobe direction and, consequently, it derives from a higher energy bin with index $i<l$. Mathematically, the threshold $\xi_{l s}^{x}$ is given by

$$
\xi_{l s}^{x}=\arg \max _{(i<l)}\left(\nu_{l i} \cdot \mathcal{N}_{i s}\right)+\xi^{z}, \quad l \geq 2
$$

where $\mathcal{N}_{i s}$ is the $i$ th element in (14), and $\nu_{l i}$ is a scaling factor which puts in relation the antenna gain in the $b_{l s}$ th and $b_{i s}$ th steering directions in the form

$$
\nu_{l i}=\left[\frac{G_{\left(b_{l s}-b_{i s}\right)}}{G_{\max }}\right]^{2}
$$

with $G_{k}$ being the array gain in the $k$ th direction, $k=$ $-N_{\text {steer }} / 2, \ldots, 0, \ldots, N_{\text {steer }} / 2$ and $G_{\left(b_{l s}-b_{i s}\right)}$ gives the array gain in the side-lobe direction with respect to the steering direction, as shown in the left of Fig. 1 . Note that $G_{0}=G_{\max }$ is the maximum array gain.

Notably, the threshold design does not require a-priori knowledge of the environment but it operates directly on the measured data. In fact, $\xi_{l s}^{x}$ is evaluated for each time bin $s$ according to the measured environment, the steering angles and the antenna gain in that directions. As stated before, this approach neglects the effect of possible false bin classification

\footnotetext{
${ }^{6}$ Such a procedure is possible thanks to the high difference of the main beam and the side-lobes in the considered massive arrays. Moreover, the constructive interference deriving from multiple targets can be accounted for by introducing an extra marging in the threshold.
} 


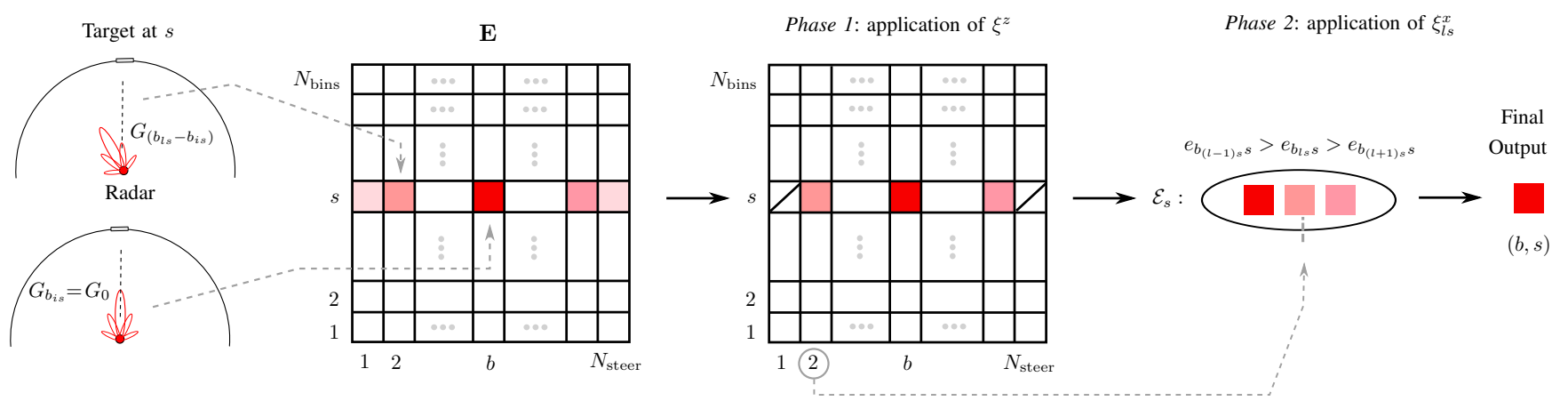

Fig. 1. Example of beamsteering scheme, with the construction of $\mathbf{E}$ and the two thresholding phases.

when consecutive targets constructively overlap due to sidelobes but, as shown in numerical results, such an effect does not impact too much on the map accuracy due to the high difference between the main beam and the side-lobes.

\section{RESUlts}

To validate the previously described system, we now exploit as a case study the mm-wave measurement campaign extensively analyzed in [20]. In particular, measurements were collected centered at $60 \mathrm{GHz}$ with $6 \mathrm{GHz}$ bandwidth, and transmitarrays (TAs) with 400 elements and only one bit for phase compensation [2] were adopted. Such arrays represent a practical solution when array complexity has to be kept as low as possible while preserving good directional radiation properties. To meet the requirements of the considered mobile radar, 37 steering directions were considered ranging from $-90^{\circ}$ to $+90^{\circ}$ with a step of $5^{\circ}$. Measurements were conducted in two indoor environments, that is an office room and a corridor. They were chosen due to their different backscattering characteristics, since the room is dense of furniture, whereas the corridor can be considered as a furniture-free and tunnelshaped environment, with a completely different geometry. As transmitted signal, root raised cosine (RRC) pulses, centered in the same bandwidth, have been considered.

Thresholds have been set by using (6) and (16) and accounting for the non-idealities of the measurements. ${ }^{7}$ In order to have a quantitative comparison among the approaches, indicate with $\mathbf{m}$ and $\mathbf{m}_{\text {ref }}$ the reconstructed and the reference map, respectively, both divided in bins/pixels. Then, we define the map similarity score metric between the two maps as

$$
\Psi\left(\mathbf{m}, \mathbf{m}_{\mathrm{ref}}\right)=d\left(\mathbf{m}, \mathbf{m}_{\mathrm{ref}}, o\right)+d\left(\mathbf{m}_{\mathrm{ref}}, \mathbf{m}, o\right)
$$

with

$$
d\left(\mathbf{m}, \mathbf{m}_{\mathrm{ref}}, o\right)=\frac{\sum_{q \in \mathbf{q}_{o}} \min \left(\operatorname{dist}\left(q, \mathbf{q}_{\mathrm{ref}}\right)\right)}{N_{o}}
$$

where $o$ denotes the occupied state of the bin, $N_{o}$ is the number of occupied bins in $\mathbf{m}, \mathbf{q}_{o}=[(x, y) \mid \mathbf{m}(x, y)=o]$ denotes the vector of the spatial coordinates $(x, y)$ such that the correspondent bin is occupied, and $\mathbf{q}_{\mathrm{ref}}=\left[(x, y) \mid \mathbf{m}_{\mathrm{ref}}(x, y)=o\right]$

\footnotetext{
${ }^{7}$ The threshold accounts for a margin to mask the impairments due to the mechanical beamsteering. In fact, if from one side this operation preserves the same pattern in all the directions, small misalignments were present due to some mechanical issues, but they did not affect the measurement validity.
}

indicates the counterpart in the reference map. Thus, the considered metric allows to have a quantitative evaluation of how much the obtained map differs from the initial one. Note that, ideally, in case of perfect map reconstruction, $\Psi\left(\mathbf{m}, \mathbf{m}_{\text {ref }}\right)=0$. Obviously, the considered approach suffers from the not perfect reproduction of the environment, but it represents a reliable benchmark for performance comparison. Differently from [21], for each steering direction $\theta_{b}$, $\operatorname{dist}\left(q, \mathbf{q}_{\mathrm{ref}}\right)$ accounts for the distance in terms of number of bins/pixels of the two maps along $\theta_{b}$. Obviously, this approach neglects the estimate of the energy level but gives a further indicator of the map quality with respect to the visual inspection.

Figure 2 shows the true $\mathbf{m}_{\text {ref }}$ map (e.g., a mere reproduction of the true layout) and the maps estimated at the output of phase 1 and 2 for both the corridor and the office room, respectively. The value 0 is assigned to not-occupied state of the bin in the estimated map. As it can be seen, results are promising as with this simple approach the map is cleaned. ${ }^{8}$ Thanks to the adaptive threshold, the similarity score is improved of approximately the $36 \%$ in corridor and the $78 \%$ in the room, with respect to the noise threshold. Thus, it is experimentally verified, that our algorithm, designed for the detection of a unique target, still performs well in environments showing different backscattering characteristics, thanks to the adoption of extremely narrow beam arrays. This further motivates the importance of adopting massive instead of non-massive arrays.

Fig. 3 reports the mapping reconstruction when the radar is located in a different position of the room as measurements were collected each $0.5 \mathrm{~m}$, to show the robustness of the algorithm also when moving in an indoor environment.

Considering the comparison with other indoor mapping techniques, those relying on coherent receivers, digital beamforming and more complex algorithms are expected to attain better performance but, due to the entailed complexity in their architectures, they are not suitable for personal radars applications. Furthermore, by properly tuning the algorithm parameters in the extended version of the CLEAN approach described in [20], it is possible to attain a very defined environment contour. Unfortunately, to practically realize the method, there is the need to adopt high-cost hardware to

${ }^{8}$ Differences in the reconstruction of the true perimeter layout can be ascribed also to possible imperfections in the environment representation in simulations, and in neglecting the presence of furniture, since it would require a precision level which is out of the scope of the manuscript. 

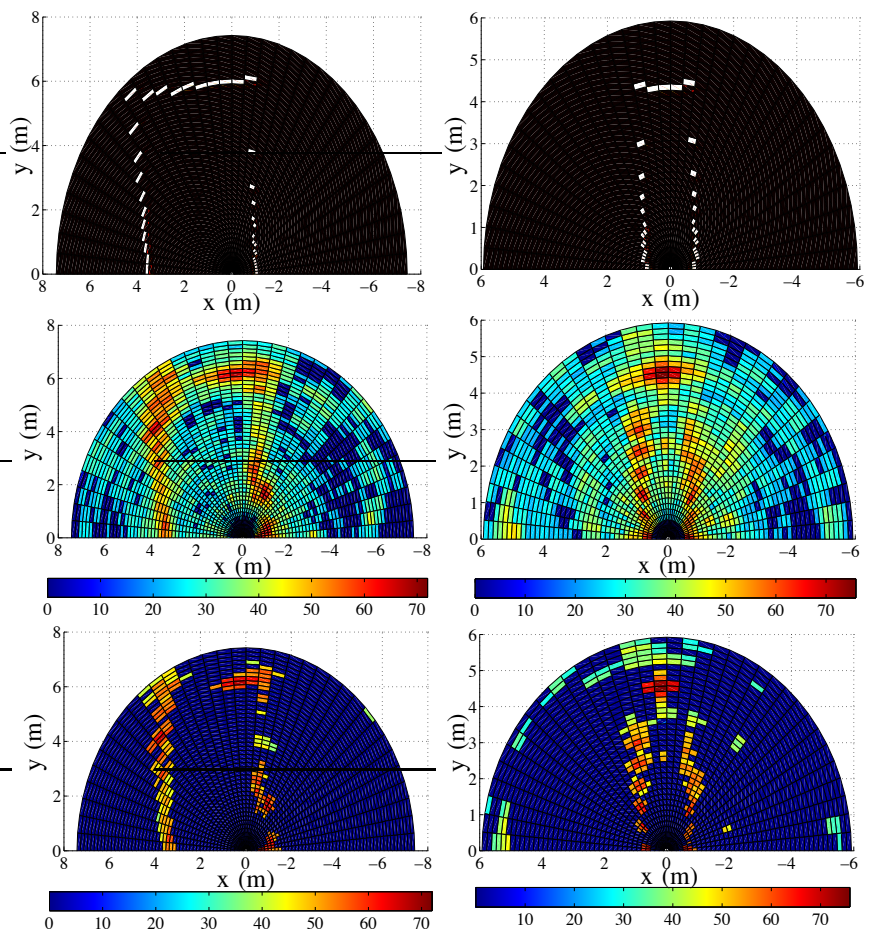

Fig. 2. Mapping results in the office room (left) and in the corridor (right) Top: Reference map $\mathbf{m}_{\text {ref }}$ where white bins indicate the environment to be reconstructed. Middle: Estimated map $\mathbf{m}$ at the output of phase 1. Bottom Estimated map $\mathbf{m}$ at the output of phase 2.
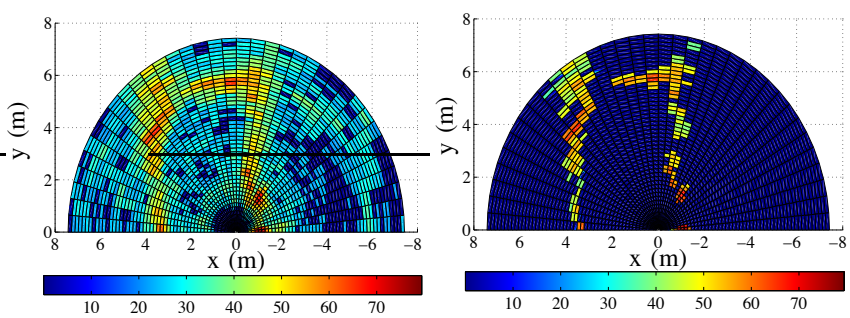

Fig. 3. Left: Estimated map $\mathbf{m}$ at the output of phase 1. Right: Estimated map $\mathbf{m}$ at the output of phase 2. Results are collected from a different radar position obtained by moving the radar of $0.5 \mathrm{~m}$ along the $y$-axe.

guarantee the perfect signals alignment required by CLEAN. On the other side, in [5], preliminary interesting results using a low-complexity receiver are reported. Unfortunately, as previously stated, such an approach is limited by the need to perform a preliminary characterization of the backscattering characteristics of the surrounding environment, which might be not possible in real-time applications. Thus, the solution herein proposed can ease the practical integration in radar transceivers with a simple and environment-adaptive scheme. To further clean the map, our method can be refined by discarding, as an example, components containing a low percentage of the overall measured energy, as typically done in CLEAN-inspired algorithms.

\section{CONCLuSions}

In this paper we proposed the design of a radar, suitable for small-size and low-complexity devices, capable of self- estimating a threshold for cleaning measured data during the beamsteering operation without requiring a-priori information of the environment. We showed the feasibility of the proposed approach by exploiting measurements collected with real 400element massive arrays at mm-wave. It has been experimentally verified that our algorithm allows to mitigate the sidelobes effect in dense scatterers scenarios without the need of a more complex coherent receiver architecture or of more complex CLEAN-like algorithms.

\section{REFERENCES}

[1] F. Guidi, A. Guerra, and D. Dardari, "Personal mobile radars with millimeter-wave massive arrays for indoor mapping," IEEE Trans. Mobile Comp., vol. 14, no. 99, 2015.

[2] H. Kaouach et al., "Wideband low-loss linear and circular polarization transmit-arrays in V-band," IEEE Trans. Antennas Propag., vol. 59, no. 7, pp. 2513-2523, July 2011.

[3] A. Guerra, F. Guidi, and D. Dardari, "Single-anchor localization and orientation performance limits using massive arrays: MIMO vs. beamforming," IEEE Trans. Wireless Commun., pp. 1-1, 2018.

[4] S.-H. Fang and C.-H. Wang, "A dynamic hybrid projection approach for improved wi-fi location fingerprinting," IEEE Trans. Veh. Tech., vol. 60, no. 3, pp. 1037-1044, 2011.

[5] F. Guidi et al., "Joint energy detection and massive array design for localization and mapping," IEEE Trans. Wireless Commun., vol. 16, no. 3, pp. 1359-1371, Mar. 2017.

[6] D. Dardari, P. Closas, and P. M. Djurić, "Indoor tracking: Theory, methods, and technologies," IEEE Trans. Veh. Tech., vol. 64, no. 4, pp. 1263-1278, Apr. 2015.

[7] H. Deng, "Effective CLEAN algorithms for performance-enhanced detection of binary coding radar signals," IEEE Trans. Signal Process., vol. 52, no. 1, pp. 72-78, Jan 2004.

[8] J. Tsao and B. Steinberg, "Reduction of sidelobe and speckle artifacts in microwave imaging: the CLEAN technique," IEEE Trans. Antennas Propag., vol. 36, no. 4, pp. 543-556, Apr 1988.

[9] Q. H. Spencer et al., "Modeling the statistical time and angle of arrival characteristics of an indoor multipath channel," IEEE J. Sel. Areas Commun., vol. 18, no. 3, pp. 347-360, March 2000.

[10] E. Kelly, "An adaptive detection algorithm," IEEE Trans. Aerosp. Electron. Syst., vol. AES-22, no. 2, pp. 115-127, March 1986.

[11] U. Nickel, "Detection with adaptive arrays with irregular digital subarrays," in Proc. IEEE Radar Conf., April 2007, pp. 635-640.

[12] C. Richmond, "Performance of the adaptive sidelobe blanker detection algorithm in homogeneous environments," IEEE Trans. Signal Process., vol. 48, no. 5, pp. 1235-1247, May 2000.

[13] K. Sangston, F. Gini, and M. Greco, "Coherent radar target detection in heavy-tailed compound-Gaussian clutter," IEEE Trans. Aerosp. Electron. Syst., vol. 48, no. 1, pp. 64-77, Jan 2012.

[14] F. Bandiera et al., "An improved adaptive sidelobe blanker," IEEE Trans. Signal Process., vol. 56, no. 9, pp. 4152-4161, Sept 2008.

[15] W. Liu et al., "Adaptive double subspace signal detection in Gaussian background - part i: Homogeneous environments," IEEE Trans. Signal Process., vol. 62, no. 9, pp. 2345-2357, May 2014.

[16] L. Jing et al., "Design and Performance Analysis of Noncoherent Detection Systems With Massive Receiver Arrays." IEEE Trans. Signal Processing, vol. 64, no. 19, pp. 5000-5010, 2016.

[17] X. Gao et al., "Energy-Efficient Hybrid Analog and Digital Precoding for MmWave MIMO Systems With Large Antenna Arrays," IEEE J. Sel. Areas Commun., vol. 34, no. 4, pp. 998-1009, Apr. 2016.

[18] W. Hong et al., "Study and prototyping of practically large-scale mmwave antenna systems for 5G cellular devices," IEEE Commun. Mag., vol. 52, no. 9, pp. 63-69, 2014.

[19] A. Mariani, A. Giorgetti, and M. Chiani, "Effects of noise power estimation on energy detection for cognitive radio applications," IEEE Trans. Commun., vol. 59, no. 12, pp. 3410-3420, Dec. 2011.

[20] A. Guerra et al., "A millimeter-wave indoor backscattering channel model for environment mapping," IEEE Trans. Antennas Propag., vol. 65, no. 9, pp. 4935-4940, Sept 2017.

[21] I. Varsadan, A. Birk, and M. Pfingsthorn, "Determining map quality through an image similarity metric," in Robot Soccer World Cup. Springer, 2008, pp. 355-365. 